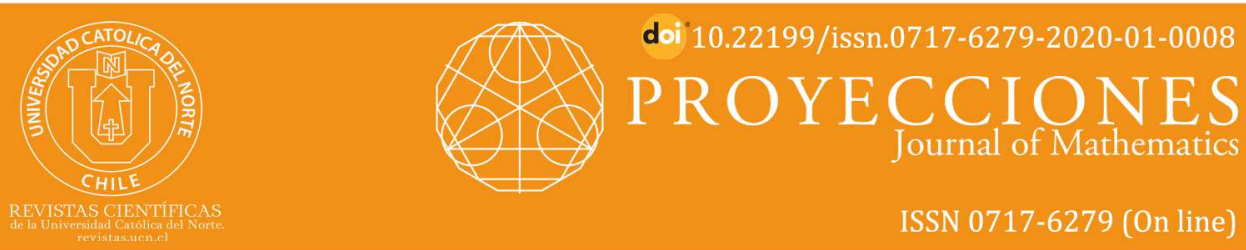

\title{
Weak convergence and weak compactness in the space of integrable functions with respect to a vector measure
}

Charles Swartz ${ }^{1}$

${ }^{1}$ New Mexico State University, Mathematics Dept, Las Cruces, NM, U. S. A.

-cswartz@nmsu.edu

Received: December 2018 | Accepted: August 2019

\section{Abstract:}

We consider weak convergence and weak compactness in the space $L^{1}(\mathrm{~m})$ of real valued integrable functions with respect to a Banach space valued measure $m$ equipped with its natural norm. We give necessary and sufficient conditions for a sequence in $L^{1}(\mathrm{~m})$ to be weak Cauchy, and we give necessary and sufficient conditions for a subset of $L^{1}(\mathrm{~m})$ to be conditionally sequentially weakly compact.

Keywords: Weak convergence; Weak compactness; Integrable functions; Measure and integration.

MSC (2010): 28B15; 28B20.

\section{Cite this article as (IEEE citation style):}

C. Swartz, "Weak convergence and weak compactness in

the space of integrable functions with respect to a vector measure", Proyecciones (Antofagasta, On line), vol. 39, no. 1, pp. 123-133, Feb. 2020, doi: 10.22199/issn.07176279-2020-01-0008. [Accessed dd-mm-yyyy]

Article copyright: (C) 2020 Charles Swartz. This is an open access article distributed under the terms of the Creative Commons Licence, which permits unrestricted use and distribution provided the original author and source are credited.

(cc) BY 


\section{Introduction}

In this note we consider weak convergence and weak compactness in the space, $L^{1}(m)$, of integrable functions with respect to a Banach space valued, countably additive measure $m$. We use a characterization of the dual space $L^{1}(m)^{\prime}$ due to Okada $([7])$.

Let $\Sigma$ be a $\sigma$-algebra of subsets of a set $S$, let $X$ be a Banach space and $m: \Sigma \rightarrow X$ be countably additive. We consider the space $L^{1}(m)$ of functions $f: S \rightarrow \mathbf{R}$ which are integrable with respect to $m$. A measurable function $f: S \rightarrow \mathbf{R}$ is weakly $m$ integrable if $f$ is $x^{\prime} m=x^{\prime} \circ m$ integrable for every $x^{\prime} \in X^{\prime}$ and $f$ is $m$ integrable if $f$ is weakly $m$ integrable and for every $A \in \Sigma$ there exists $x_{A} \in X$ such that $\int_{A} f d x^{\prime} m=x^{\prime}\left(x_{A}\right)$ for $x^{\prime} \in X^{\prime}$; We set $x_{A}=\int_{A} f d m$. (See [5],[6] for the properties of the integral; another approach to the integral is given in [2] IV.10.) This space is a Banach space with respect to the norm

$$
\|f\|_{1}=\sup \left\{\int_{S}|f| d\left|x^{\prime} m\right|:\left\|x^{\prime}\right\| \leq 1\right\}
$$

where $\left|x^{\prime} m\right|$ denotes the variation of the scalar measure $x^{\prime} m$.

Notation 1. If $g \in L^{\infty}(m), A \in \Sigma$ and $x^{\prime} \in X^{\prime}$, denote the continuous linear functional

$$
f \rightarrow \int_{S} g f \chi_{A} d x^{\prime} m=\int_{A} g f d x^{\prime} m
$$

on $L^{1}(m)$ by $g \chi_{A} x^{\prime}$.

Okada has shown that if $l \in L^{1}(m)^{\prime}$ there exist $g \in L^{\infty}(m),\left\{A_{j}\right\} \subset \Sigma$ pairwise disjoint and $x_{j}^{\prime} \in X^{\prime},\left\|x_{j}^{\prime}\right\| \leq 1$, such that

$$
l(f)=\sum_{j=1}^{\infty} \int_{A_{j}} g f d x_{j}^{\prime} m
$$

for $f \in L^{1}(m)$, where the series converges; that is, $l=\sum_{j=1}^{\infty} g \chi_{A_{j}} x_{j}^{\prime}([7])$. Note that if the series $\sum_{j=1}^{\infty} \int_{A_{j}} g f d x_{j}^{\prime} m$ converges for every $f \in L^{1}(m)$ when $g \in L^{\infty}(m),\left\{A_{j}\right\} \subset \Sigma$ pairwise disjoint and $x_{j}^{\prime} \in X^{\prime},\left\|x_{j}^{\prime}\right\| \leq 1$, then $l(f)=\sum_{j=1}^{\infty} \int_{A_{j}} g f d x_{j}^{\prime} m$ defines a continuous linear functional on $L^{1}(m)($ for each $n, l_{n}(f)=\sum_{j=1}^{n} \int_{A_{j}} g f d x_{j}^{\prime} m$ is linear and continuous and 


$$
l_{n}(f) \rightarrow \sum_{j=1}^{\infty} \int_{A_{j}} g f d x_{j}^{\prime} m=l(f)
$$

for every $f$ so $l$ is continuous by the Banach-Steinhaus Theorem).

Thus, the dual of $L^{1}(m)$ is the space of all $g \in L^{\infty}(m),\left\{A_{j}\right\} \subset \Sigma$ pairwise disjoint and $x_{j}^{\prime} \in X^{\prime},\left\|x_{j}^{\prime}\right\| \leq 1$, such that the series $\sum_{j=1}^{\infty} \int_{A_{j}} g f d x_{j}^{\prime} m$ converges for every $f \in L^{1}(m)$.

Not every $g \in L^{\infty}(m),\left\{A_{j}\right\} \subset \Sigma$ pairwise disjoint and $x_{j}^{\prime} \in X^{\prime},\left\|x_{j}^{\prime}\right\| \leq$ 1 , may define elements of $L^{1}(m)^{\prime}$; that is, there may exist such elements where the series $\sum_{j=1}^{\infty} \int_{A_{j}} g f d x_{j}^{\prime} m$ may fail to converge. Indeed, we have

Theorem 2. The measure $m$ has bounded variation iff the series $\sum_{j=1}^{\infty} \int_{A_{j}} g f d x_{j}^{\prime} m$ converges for every $f \in L^{1}(|m|), g \in L^{\infty}(m),\left\{A_{j}\right\} \subset \Sigma$ pairwise disjoint and $x_{j}^{\prime} \in X^{\prime},\left\|x_{j}^{\prime}\right\| \leq 1$.

Proof. Suppose $m$ has bounded variation, $|m|$. If $f \in L^{1}(|m|), g \in$ $L^{\infty}(m),\left\{A_{j}\right\} \subset \Sigma$ pairwise disjoint and $x_{j}^{\prime} \in X^{\prime},\left\|x_{j}^{\prime}\right\| \leq 1$, then

$$
\begin{aligned}
\left|\sum_{j=1}^{\infty} \int_{A_{j}} g f d x_{j}^{\prime} m\right| & \leq \sum_{j=1}^{\infty}\left|\int_{A_{j}} g f d x_{j}^{\prime} m\right| \leq \sum_{j=1}^{\infty} \int_{A_{j}}|g f| d\left|x_{j}^{\prime} m\right| \\
& \leq \sum_{j=1}^{\infty} \int_{A_{j}}|g f| d|m| \leq\|g\|_{\infty} \sum_{j=1}^{\infty} \int_{A_{j}}|f| d|m| \\
& \leq\|g\|_{\infty} \int_{S}|f| d|m|
\end{aligned}
$$

shows the series converges.

For the converse, set $f=g=1$ and let $\left\{A_{j}\right\}$ be any pairwise disjoint sequence from $\Sigma$. Pick $x_{j}^{\prime} \in X^{\prime},\left\|x_{j}^{\prime}\right\| \leq 1$, such that $x_{j}^{\prime}\left(m\left(A_{j}\right)\right)=\left\|m\left(A_{j}\right)\right\|$. Then

$$
\sum_{j=1}^{\infty} \int_{A_{j}} g f d x_{j}^{\prime} m=\sum_{j=1}^{\infty} x_{j}^{\prime}\left(m\left(A_{j}\right)\right)=\sum_{j=1}^{\infty}\left\|m\left(A_{j}\right)\right\|<\infty .
$$

This implies $m$ has bounded variation ([12],[10] 3.51).

The computation above shows that if $m$ has bounded variation and $L^{1}(m)=L^{1}(|m|)$, then the dual of $L^{1}(m)$ is the space of all $g \in L^{\infty}(m)$, 
$\left\{A_{j}\right\} \subset \Sigma$ pairwise disjoint and $x_{j}^{\prime} \in X^{\prime},\left\|x_{j}^{\prime}\right\| \leq 1$, such that the series $\sum_{j=1}^{\infty} \int_{A_{j}} g f d x_{j}^{\prime} m$ converges for every $f \in L^{1}(m)$.

For later use we observe that if $l=\sum_{j=1}^{\infty} g \chi_{A_{j}} x_{j}^{\prime} \in L^{1}(m)^{\prime}$, then the series $\sum_{j=1}^{\infty} \int_{A_{j}} g f d x_{j}^{\prime} m$ is subseries convergent for every $f \in L^{1}(m)$. Indeed, if $\sigma \subset \mathbf{N}$ and $f \in L^{1}(m)$ let $A=\cup_{j \in \sigma} A_{j}$ and set $f_{\sigma}=\chi_{A} f \in L^{1}(m)$. Then

$$
l\left(f_{\sigma}\right)=\sum_{j=1}^{\infty} \int_{A_{j}} g f_{\sigma} d x_{j}^{\prime} m=\sum_{j \in \sigma} \int_{A_{j}} g f d x_{j}^{\prime} m .
$$

We now consider weak convergence and weak sequential compactness in $L^{1}(m)$. First we consider necessary conditions for weak sequential compactness. A subset $K$ of $L^{1}(m)$ is relatively sequentially weak compact if every sequence $\left\{f_{k}\right\}$ in $K$ has a subsequence which is weakly convergent and $K$ is conditionally sequentially weakly compact if every sequence $\left\{f_{k}\right\}$ in $K$ has a subsequence which is weakly Cauchy.

Theorem 3. Suppose $K \subset L^{1}(m)$ is conditionally sequentially weakly compact. Then

(\#) for every $\sum_{j=1}^{\infty} g \chi_{A_{j}} x_{j}^{\prime} \in L^{1}(m)^{\prime}$, the series $\left\{\sum_{j=1}^{\infty} \int_{A_{j}} g f d x_{j}^{\prime} m: f \in\right.$ $K\}$ are uniformly subseries convergent.

Proof. If the conclusion fails to hold, there exist $\epsilon>0, f_{k} \in K$ and an increasing sequence of intervals $\left\{I_{k}\right\}$ with

$$
(*)\left|\sum_{j \in I_{k}} \int_{A_{j}} g f_{k} d x_{j}^{\prime} m\right|>\epsilon .
$$

We may assume that $\lim _{k}\left\langle l, f_{k}\right\rangle$ exists for every $l \in L^{1}(m)^{\prime}$. For any $\sigma \subset \mathbf{N}$, define $l_{\sigma}$ belonging to $L^{1}(m)^{\prime}$ by

$$
\left\langle l_{\sigma}, f\right\rangle=\sum_{j \in \sigma} \int_{A_{j}} g f d x_{j}^{\prime} m .
$$

Then for every $\sigma \subset \mathbf{N}$,

$$
\lim _{k}\left\langle l_{\sigma}, f_{k}\right\rangle=\lim _{k} \sum_{j \in \sigma} \int_{A_{j}} g f_{k} d x_{j}^{\prime} m
$$

exists. Set $a_{j}=\lim _{k} \int_{A_{j}} g f_{k} d x_{j}^{\prime} m$. By the Hahn-Schur Theorem ([10]7.18), the series 


$$
\left\{\sum_{j=1}^{\infty} \int_{A_{j}} g f_{k} d x_{j}^{\prime} m: k\right\}
$$

are uniformly subseries convergent and

$$
\lim _{k} \sum_{j \in \sigma} \int_{A_{j}} g f d x_{j}^{\prime} m=\sum_{j \in \sigma} a_{j}
$$

uniformly for $\sigma \subset \mathbf{N}$. But, this contradicts $(*)$.

Remark 4. Note that the conclusion (\#) in Theorem 3 implies that the indefinite integrals $\left\{\int . g f d m: f \in K\right\}$ are uniformly countably additive. For if this is not the case, there exist $\epsilon>0$, pairwise disjoint $\left\{A_{j}\right\} \subset \Sigma, f_{k} \in K$ and an increasing sequence of intervals $\left\{I_{k}\right\}$ such that $\left\|\sum_{j \in I_{k}} \int_{A_{j}} g f_{k} d m\right\|>$ $\epsilon$. Set $B_{k}=\cup_{j \in I_{k}} A_{j}$ and pick $x_{k}^{\prime} \in X^{\prime},\left\|x_{k}^{\prime}\right\| \leq 1$, such that

$$
\left|x_{k}^{\prime} \sum_{j \in I_{k}} \int_{A_{j}} g f_{k} d m\right|=\left|\int_{B_{k}} g f_{k} d x_{k}^{\prime} m\right|=\left\|\sum_{j \in I_{k}} \int_{A_{j}} g f_{k} d m\right\|>\epsilon .
$$

Using $g,\left\{B_{k}\right\},\left\{x_{k}^{\prime}\right\}$ this contradicts the condition (\#).

Theorem 5. Suppose $K \subset L^{1}(m)$ is conditionally (relatively) sequentially weakly compact. Then

(\#\#) for every $g \in L^{\infty}(m), A \in \Sigma$ the set $\left\{\int_{A} g f d m: f \in K\right\}$ is conditionally (relatively) sequentially weakly compact.

Proof. The integration map $I_{g}: L^{1}(m) \rightarrow X, I_{g}(f)=\int_{A} g f d m$, is norm continuous

$$
\begin{aligned}
\left(\left\|I_{g} f\right\|\right. & =\sup \left\{\left|\int_{S} g f d x^{\prime} m\right|:\left\|x^{\prime}\right\| \leq 1\right\} \\
& \left.\leq\|g\|_{\infty} \sup \left\{\int_{S}|f| d\left|x^{\prime} m\right|:\left\|x^{\prime}\right\| \leq 1\right\}=\|g\|_{\infty}\|f\|_{1}\right)
\end{aligned}
$$

and, therefore, weak-weak continuous so the result is immediate.

We next consider sufficient conditions for weak sequential compactness. First an observation about weak convergence. 
Lemma 6. Let $\left\{f_{k}\right\} \subset L^{1}(m)$. Then $\left\{f_{k}\right\}$ is weakly Cauchy iff for every $\sum_{j=1}^{\infty} g \chi_{A_{j}} x_{j}^{\prime} \in L^{1}(m)^{\prime}$

(i) $\lim _{k} \int_{A_{j}} g f_{k} d x_{j}^{\prime} m=a_{j}$ exists for every $j$ and

(ii) the series $\left\{\sum_{j=1}^{\infty} \int_{A_{j}} g f_{k} d x_{j}^{\prime} m: k \in \mathbf{N}\right\}$ converge uniformly.

Proof. $\quad \Rightarrow$ : For (i) consider the linear functional $l_{j}=g \chi_{A_{j}} x_{j}^{\prime} \in L^{1}(m)^{\prime}$. For (ii) consider the linear functional

$$
l_{\sigma}=\sum_{j=1}^{\infty} g \chi_{A_{j}} x_{j}^{\prime} \in L^{1}(m)^{\prime}, \sigma \subset \mathbf{N} .
$$

Since $\lim _{k} l_{\sigma}(f)=\lim _{k} \sum_{j \in \sigma} \int_{A_{j}} g f_{k} d x_{j}^{\prime} m$ exists, the Hahn-Schur Theorem ([10] 7.18) gives (ii) and also implies $\lim _{k} \sum_{j \in \sigma} \int_{A_{j}} g f_{k} d x_{j}^{\prime} m=\sum_{j \in \sigma} a_{j}$ uniformly for $\sigma \subset \mathbf{N}$.

$\Leftarrow$ : Let $\epsilon>0, l=\sum_{j=1}^{\infty} g \chi_{A_{j}} x_{j}^{\prime} \in L^{1}(m)^{\prime}$. By (ii) there exists $N$ such that $\left|\sum_{j=N+1}^{\infty} \int_{A_{j}} g f_{k} d x_{j}^{\prime} m\right|<\epsilon$ for all $k$. By (i) there exists $k_{0}$ such that $i, k \geq k_{0}$ implies

$$
\left|\sum_{j=1}^{N} \int_{A_{j}} g f_{k} d x_{j}^{\prime} m-\sum_{j=1}^{N} \int_{A_{j}} g f_{i} d x_{j}^{\prime} m\right|<\epsilon .
$$

Then

$$
\begin{aligned}
& \left|l\left(f_{k}\right)-l\left(f_{i}\right)\right| \leq\left|\sum_{j=1}^{N} \int_{A_{j}} g f_{k} d x_{j}^{\prime} m-\sum_{j=1}^{N} \int_{A_{j}} g f_{i} d x_{j}^{\prime} m\right| \\
& +\left|\sum_{j=N+1}^{\infty} \int_{A_{j}} g f_{k} d x_{j}^{\prime} m\right|+\left|\sum_{j=N+1}^{\infty} \int_{A_{j}} g f_{i} d x_{j}^{\prime} m\right|<3 \epsilon
\end{aligned}
$$

for $i, k \geq k_{0}$.

Remark 7. Note the proof above implies that $f_{k} \rightarrow 0$ weakly iff (i) holds for $a_{j}=0$ and (ii).

We consider conditional sequential weak compactness.

Theorem 8. If $K \subset L^{1}(m)$ satisfies conditions (\#) and (\#\#), then $K$ is conditionally sequentially weakly compact. 
Proof. Let $\left\{f_{k}\right\} \subset K$. There exists a countable algebra $\mathcal{A}$ such that each $f_{k}$ is measurable with respect to the $\sigma$ algebra $\Sigma_{1}$ generated by $\mathcal{A}$. By replacing $\Sigma$ by $\Sigma_{1}$ we may assume that $\Sigma$ is generated by a countable algebra $\mathcal{A}$. By (\#\#) and the diagonalization method, there is a subsequence of $\left\{f_{k}\right\}$, still denoted by $\left\{f_{k}\right\}$, such that

$$
\text { weak }-\lim _{k} \int_{A} f_{k} d m=F(A)
$$

exists for every $A \in \mathcal{A}$ ([4], page 238).

We claim that weak $-\lim _{k} \int_{A} f_{k} d m=F(A)$ exists for every $A \in \Sigma$. For this, put

$$
\Sigma_{1}=\left\{A \in \Sigma: \text { weak }-\lim _{k} \int_{A} f_{k} d m=F(A) \text { exists }\right\} .
$$

Note $\mathcal{A}$ is contained in $\Sigma_{1}$. We claim that $\Sigma_{1}$ is a monotone class. Suppose $B_{j} \in \Sigma_{1}$ with $B_{j} \uparrow B$. For every $j$,

$$
\text { weak }-\lim _{k} \int_{B_{j}} f_{k} d m=F\left(B_{j}\right) \in X
$$

exists. By (\#)

$$
\text { weak }-\lim _{j} \int_{B_{j}} f_{k} d m=\int_{B} f_{k} d m
$$

uniformly for $k \in \mathbf{N}$. Let $x^{\prime} \in X^{\prime}$. Then

$$
\lim _{k} x^{\prime} \int_{B_{j}} f_{k} d m=\lim _{k} \int_{B_{j}} f_{k} d x^{\prime} m=x^{\prime} F\left(B_{j}\right)
$$

for all $j$ and

$$
\lim _{j} x^{\prime} \int_{B_{j}} f_{k} d m=x^{\prime} F\left(B_{j}\right)=\lim _{j} \int_{B_{j}} f_{k} d x^{\prime} m=x^{\prime} \int_{B} f_{k} d m=\int_{B} f_{k} d x^{\prime} m
$$

uniformly for $k \in \mathbf{N}$. By the Iterated Limit Theorem ([2] I.7.6),

$$
\lim _{j} \lim _{k} \int_{B_{j}} f_{k} d x^{\prime} m=\lim _{k} \lim _{j} \int_{B_{j}} f_{k} d x^{\prime} m=\lim _{j} x^{\prime} F\left(B_{j}\right)=\lim _{k} \int_{B} f_{k} d x^{\prime} m .
$$

Therefore, $\left\{\int_{B} f_{k} d m\right\}_{k}$ is weak Cauchy. But (\#\#) implies $\left\{\int_{B} f_{k} d m\right\}_{k}$ is relatively weak compact so weak $-\lim _{k} \int_{B} f_{k} d m=F(B)$ exists. Hence, 
$B \in \Sigma_{1}$ and $\Sigma_{1}$ is a monotone class. By the Monotone Class Theorem ([3] I.6, [9] 2.1.6) $\Sigma_{1}=\Sigma$ and the claim is established.

Thus, weak - $\lim _{k} \int_{S} g f_{k} d m$ exists for every simple function $g$.

We next claim that $\left\{\int_{S} g f_{k} d m\right\}$ is weak Cauchy for every $g \in L^{\infty}(m)$. Let $\epsilon>0$. Pick a simple function $h$ such that $\|h-g\|_{\infty}<\epsilon$. Fix $x^{\prime} \in X^{\prime}$, $\left\|x^{\prime}\right\| \leq 1$. Then

$$
\begin{aligned}
& \text { (\&) } \mid x^{\prime}\left(\int_{S} g\left(f_{k}-f_{j}\right) d m \mid\right.=\left|\int_{S} g\left(f_{k}-f_{j}\right) d x^{\prime} m\right| \\
& \leq \int_{S}(g-h)\left(f_{k}-f_{j}\right) d m|+| \int_{S} h\left(f_{k}-f_{j}\right) d m \mid \\
& \leq\|g-h\|_{\infty} \int_{S}\left|f_{k}-f_{j}\right| d\left|x^{\prime} m\right|+\left|\int_{S} h\left(f_{k}-f_{j}\right) d m\right| \\
& \leq \epsilon\left\|f_{k}-f_{j}\right\|_{1}+\left|\int_{S} h\left(f_{k}-f_{j}\right) d m\right| .
\end{aligned}
$$

The first term on the right hand side of $(\&)$ is bounded by some $M \epsilon>0$ and the last term is small for $j, k$ large by the result for simple functions established above and the claim is established.

By $(\# \#)\left\{\int_{S} g f_{k} d m\right\}$ is relatively sequentially weak compact so

$$
(*) \text { weak }-\lim _{k} \int_{S} g f_{k} d m
$$

exists.

Let $l=\sum_{j=1}^{\infty} g \chi_{B_{j}} x_{j}^{\prime} \in L^{1}(m)^{\prime}$. We show $\lim _{k} l\left(f_{k}\right)$ exists and this will establish the result. By $(*)$

$$
\lim _{k} \int_{B_{j}} g f_{k} d x_{j}^{\prime} m=\lim _{k} x_{j}^{\prime} \int_{B_{j}} g f_{k} d m
$$

exists for every $j$ and by (\#) the series $\left\{\sum_{j=1}^{\infty} \int_{B_{j}} g f_{k} d x_{j}^{\prime} m\right\}_{k}$ converge uniformly for $k \in \mathbf{N}$. Lemma 6 shows $\lim _{k} l\left(f_{k}\right)$ exists.

Another similar weak compactness result for a "weak type topology" on $L^{1}(m)$ is established in [11] 9.15 .

If $L^{1}(m)$ is weakly sequentially complete, the conditions (\#) and (\#\#) above in Theorem 8 imply that the subset $K$ is relatively sequentially compact. Conditions which guarantee that $L^{1}(m)$ is order isomorphic to an AL space and is weakly sequentially complete are given in [1] and conditions 
for which $L^{1}(m)$ is reflexive and is, therefore, weakly sequentially complete are given in [7]. See also [8].

We use the results above to show that $L^{1}(m)$ is weakly sequentially complete when $m$ is an atomic measure and $X$ is weakly sequentially complete.

Let $\mathcal{P}$ be the power set of $\mathbf{N}$ and let $m: \mathcal{P} \rightarrow X$ be countably additive. First, an observation.

Proposition 9. The function $f: \mathbf{N} \rightarrow \mathbf{R}$ is $m$ integrable $\Longleftrightarrow$ the series $\sum_{j=1}^{\infty} f(j) m(j)$ is subseries convergent.

Proof. $\Longrightarrow$ : Follows from the countable additivity of the integral $\int f d m$. $\Longleftarrow$ : Let $x^{\prime} \in X^{\prime}$ and $A \in \mathcal{P}$. Then $\int_{A} f d x^{\prime} m=\sum_{j \in A} f(j) x^{\prime} m(j)=x^{\prime}$ $\sum_{j \in A} f(j) m(j)$ so $f$ is $m$ integrable with $\int_{A} f d m=\sum_{j \in A} f(j) m(j)$.

Theorem 10. If $X$ is weakly sequentially complete, then $L^{1}(m)$ is weakly sequentially complete.

Proof. Let $\left\{f_{j}\right\}$ be weakly Cauchy in $L^{1}(m)$. For $x^{\prime} \in X^{\prime}$ and $k \in$ $\mathbf{N}$, define $e^{k} x^{\prime} \in L^{1}(m)^{\prime}$ by $e^{k} x^{\prime}(f)=f(k) x^{\prime} m(k)$. Then $\lim _{j} e^{k} x^{\prime}\left(f_{j}\right)=$ $\lim _{j} x^{\prime} f_{j}(k) m(k)$ exists. Pick $x_{k}^{\prime \prime}$ such that $x_{k}^{\prime}(m(k))=\|m(k)\|$ and $\left\|x_{k}^{\prime}\right\| \leq$ 1. Define

$$
F(k)=\lim _{j} x_{k}^{\prime} f_{j}(k) m(k)=\lim _{j} f_{j}(k)\|m(k)\|
$$

and set $f(k)=F(k) /\|m(k)\|$ if $m(k) \neq 0$ and $f(k)=0$ otherwise. Then

$$
\lim _{j} f_{j}(k)=\lim _{j}\|m(k)\| f(k) /\|m(k)\|=F(k) /\|m(k)\|=f(k)
$$

so $f_{j} \rightarrow f$ pointwise.

We claim $f \in L^{1}(m)$. For $\sigma \subset \mathbf{N}$ and $x^{\prime} \in X^{\prime}$, define $l_{\sigma} \in L^{1}(m)^{\prime}$ by $l_{\sigma}(h)=\sum_{i \in \sigma} h(i) x^{\prime} m(i)$. Then $\lim _{j} l_{\sigma}\left(f_{j}\right)=\lim _{j} \sum_{i \in \sigma} f_{j}(i) x^{\prime} m(i)$ exists and $\lim _{j} f_{j}(i) x^{\prime} m(i)=f(i) x^{\prime} m(i)$. The Hahn-Schur Theorem ([10] 7.18) implies that the series $\sum_{i} f(i) x^{\prime} m(i)$ is subseries convergent and

$$
(*) \lim _{j} \sum_{i \in \sigma} f_{j}(i) x^{\prime} m(i)=\sum_{i \in \sigma} f(i) x^{\prime} m(i)
$$

uniformly for $\sigma \subset \mathbf{N}$. Thus, the series $\sum_{i} f(i) m(i)$ is weakly unconditionally Cauchy and weakly subseries convergent since $X$ is weakly sequentially 
complete. The Orlicz-Pettis Theorem gives that the series is norm subseries convergent so $f$ is $m$ integrable by the proposition above.

Next, we show $f_{j} \rightarrow f$ weakly. Let $l=\sum_{k=1}^{\infty} g \chi_{A_{k}} x_{k}^{\prime} \in L^{1}(m)^{\prime}$ with $\left\|x_{k}^{\prime}\right\| \leq 1,\left\{A_{k}\right\} \subset \mathbf{N}$ pairwise disjoint and $g \in L^{\infty}(m)$. We show $\lim _{j} l\left(f_{j}\right)$ exists by checking Lemma 6 . Now $\{g(i)\} \in l^{\infty}$. Since $X$ is weakly sequentially complete, Theorem 7.30 of [10] applies to condition $(*)$ and implies that

$$
\lim _{j} \int_{A_{k}} g f_{j} d x_{k}^{\prime}=\lim _{j} \sum_{i \in A_{k}} g(i) f_{j}(i) x_{k}^{\prime} m(i)
$$

exists for every $k$ so (i) of Lemma 6 holds. Now $\left\{f_{j}: j\right\}$ is conditionally sequentially weakly compact so condition (\#) of Theorem 3 implies (ii) of Lemma 6 . Hence, $f_{j} \rightarrow f$ weakly.

\section{Acknowledgement}

The author would like to thank Susumu Okada for his help.

\section{References}

[1] G. Curbera, "Banach space properties of $\mathrm{L}^{1}$ of a vector measure", Proceedings of the American mathematical society, vol. 123, no. 12 pp. 37973806, Dec. 1995, doi: 10.2307/2161909.

[2] N. Dunford and J. T. Schwartz, Linear operators, vol. 1. New York, NY: Interscience, 1958.

[3] P. Halmos, Measure theory, Princeton, NJ: D. Van Nostrand Company, 1950.

[4] J. Kelley, General topology, Princeton, NJ: D. Van Nostrand Company, 1955.

[5] I. Kluvanek and G. Knowles, Vector measures and control systems, Amsterdam: North Holland, 1975.

[6] D. Lewis, "Integration with respect to vector measures", Pacific journal of mathematics, vol. 33, no. 1. pp. 157-165, 1970. [On line]. Available: https://bit.ly/3138dbT

[7] S. Okada, "The dual space of $\operatorname{L1}(\mu)$ for a vector measure $\mu$ ", Journal of mathematical analysis and applications, vol. 177, no. 2, pp. 583-599, Aug. 1993, doi: 10.1006/jmaa.1993.1279. 
Weak convergence and weak compactness in the space of integrable ..133

[8] S. Okada, W. J. Ricker, E. A. Sánchez-Pérez,"Lattice copies of c0 and $\ell \infty$ in spaces of integrable functions for a vector measure", Dissertationes mathematicae, vol. 500, 2014, doi: 10.4064/dm500-0-1.

[9] C. Swartz, Measure, integration, and function spaces. Singapore: World Scientific, 1994.

[10] C. Swartz, Multiplier convergent series. Singapore: World Scientific, 2009.

[11] C. Swartz, Abstract duality pairs in analysis. Singapore: World Scientific, 2018.

[12] B. L. Thorp, "Sequential evaluation convergence", Journal of the London mathematical society, vol. s1-44, no. 1pp. 201-209, Jan. 1969, doi: 10.1112/jlms/s1-44.1.201. 